\title{
Pre-Transplant Plasma Potassium as a Potential Risk Factor for the Need of Early Hyperkalaemia Treatment after Kidney Transplantation: A Cohort Study
}

\author{
Bram C.S. de Vries Stefan P. Berger Stephan J.L. Bakker Martin H. de Borst \\ Margriet F.C. de Jong \\ Department of Internal Medicine, Division of Nephrology, University of Groningen, University Medical Center \\ Groningen, Groningen, The Netherlands
}

\section{Keywords}

Plasma potassium · Kidney transplantation · Hyperkalaemia treatment

\begin{abstract}
Introduction: Plasma potassium $\left(\mathrm{K}^{+}\right)$abnormalities are common among patients with chronic kidney disease and are associated with higher rates of death, major adverse cardiac events, and hospitalization in this population. Currently, no guidelines exist on how to handle pre-transplant plasma $\mathrm{K}+$ in renal transplant recipients (RTR). Objective: The aim of this study is to examine the relation between pre-transplant plasma $\mathrm{K}^{+}$and interventions to resolve hyperkalaemia within $48 \mathrm{~h}$ after kidney transplantation. Methods: In a singlecentre cohort study, we addressed the association between the last available plasma $\mathrm{K}^{+}$level before transplantation and the post-transplant need for dialysis or use of $\mathrm{K}^{+}$-lowering medication to resolve hyperkalaemia within $48 \mathrm{~h}$ after renal transplantation using multivariate logistic regression analysis. Results: 151 RTR were included, of whom 51 (33.8\%) patients received one or more $\mathrm{K}^{+}$interventions within $48 \mathrm{~h}$ after transplantation. Multivariate regression analysis revealed that a higher pre-transplant plasma $\mathrm{K}^{+}$was associated with
\end{abstract}

\begin{tabular}{ll}
\hline karger@karger.com & $\begin{array}{l}\odot 2020 \text { The Author(s) } \\
\text { Published by S. Karger AG, Basel }\end{array}$ \\
www.karger.com/nef & $\begin{array}{l}\text { This is an Open Access article licensed under the Creative Commons } \\
\text { Attribution-NonCommercial-4.0 International License (CC BY-NC) } \\
\text { (http://www.karger.com/Services/OpenAccessLicense), applicable to } \\
\text { the online version of the article only. Usage and distribution for com- } \\
\text { mercial purposes requires written permission. }\end{array}$
\end{tabular}

an increased risk of post-transplant intervention (odds ratio 2.2 [95\% Cl: 1.1-4.4]), independent of donor type (deceased or living) and use of $\mathrm{K}^{+}$-lowering medication within $24 \mathrm{~h}$ prior to transplantation). Conclusions: This study indicates that a higher pre-transplant plasma $\mathrm{K}^{+}$is associated with a higher risk of interventions necessary to resolve hyperkalaemia within $48 \mathrm{~h}$ after renal transplantation. Further research is recommended to determine a cutoff level for pre-transplant plasma $\mathrm{K}^{+}$that can be used in practice.

(c) 2020 The Author(s)

Published by S. Karger AG, Basel

\section{Introduction}

Chronic kidney disease (CKD) remains a major worldwide concern, affecting approximately 200-million people globally [1]. CKD may result in end-stage renal disease (ESRD), accompanied by reduced life expectancy and severely impaired quality of life [2]. Kidney transplantation is the ESRD treatment of choice and is by far the most commonly performed type of transplantation: in 2017, a total of 90,306 kidneys were transplanted worldwide $[3,4]$. However, the need for kidney donors remains 
high; for example, in the Eurotransplant region, at least 10,000 people are waiting for a kidney transplant [5]. Improving post-transplant quality of care may contribute, amongst other factors, to the reduction in post-transplant discomfort for renal transplant recipients (RTR), graft loss, morbidity, and mortality. Plasma potassium abnormalities, particularly hyperkalaemia, are common among patients with ESRD and are associated with higher rates of death, major adverse cardiac events, and hospitalization in CKD [6]. However, whether plasma potassium immediately before kidney transplantation is associated with post-transplant complications remains unknown.

In ESRD, potassium homeostasis is impaired due to a strongly reduced renal capacity to excrete sufficient amounts of potassium. Furthermore, plasma potassium may rise during the transplantation procedure, for example, due to tissue damage, administration of packed red blood cells (RBCs), and ischaemia as a result of clamping large arteries $[7,8]$. Post-transplant hyperkalaemia may lead to the need for acute dialysis and to severe complications such as cardiac arrhythmias and ICU admissions. Interventions to avoid or correct post-transplant hyperkalaemia include dialysis (both haemo- and peritoneal dialysis) and use of potassium-lowering medication, which may take place before or after transplantation. Various authors described early post-transplant hyperkalaemia rates in RTR, ranging from 18 to $80 \%[9,10]$. Weinberg et al. [10] determined that $64 \%$ of all RTR need early posttransplant hyperkalaemia treatment by haemodialysis, sodium polystyrene sulfonate, insulin/dextrose, or calcium gluconate.

To the best of our knowledge, no studies have addressed the relation between pre-transplant plasma potassium and possible post-transplant hyperkalaemia interventions after kidney transplantation. Here, we investigated the relationship between pre-transplant plasma potassium and post-transplant interventions to resolve hyperkalaemia within $48 \mathrm{~h}$ after kidney transplantation. We hypothesized that a higher pre-transplant plasma potassium is a risk factor for post-transplant interventions (potassium-lowering medication and dialysis needs) to resolve hyperkalaemia.

\section{Materials and Methods}

\section{Study Characteristics}

This cohort study was performed in the University Medical Centre Groningen (UMCG), the Netherlands. All consecutive patients who underwent kidney transplantation in the UMCG between January 1, 2014, and January 1, 2015, were included. Pa- tients younger than 18 years were excluded. Data were anonymously extracted from the hospital's digital information system, verified, and completed using information from written patient records. The Eurotransplant database was used to complete data for deceased donors. The study was approved by the institutional ethical review board (METc 2014/077). All procedures were conducted in accordance with the Declarations of Helsinki and Istanbul. During the study period, the standard immunosuppressive regimen in our centre consisted of induction treatment with basiliximab, combined with prednisolone, mycophenolate mofetil, and tacrolimus.

\section{Endpoints}

The endpoint was the occurrence of interventions to resolve hyperkalaemia within $48 \mathrm{~h}$ after kidney transplantation. Dialysis (both haemo- and peritoneal dialysis) and use of potassium-lowering medication (sodium polystyrene sulfonate, calcium polystyrene sulfonate, sodium bicarbonate, or insulin therapy for hyperkalaemia) were defined as interventions to resolve hyperkalaemia (hereafter noted as "potassium-lowering interventions"). Diuretics are not used in hyperkalaemia management in patients with ESRD in our centre and therefore were not included in our endpoint. No standard cutoff threshold or range for treating hyperkalaemia post-transplantation is defined per protocol in our centre.

\section{Demographic and Clinical Parameters}

Demographic study parameters for recipients included gender, age, and co-morbidities such as known cardiovascular characteristics and risk factors. For donors, donor type (deceased or living), gender, age, and last known serum creatinine at retrieval were collected. Deceased donors were determined as donor after brain death (DBD) or donor after cardiac death (DCD). For recipients, the number of transplantations, residual diuresis volume at admission, and dialysis status (non-dialysis-dependent chronic kidney disease or dialysis-dependent chronic kidney disease) were taken into account. Clinical parameters collected included first warm ischaemia time (WIT), cold ischaemia time (CIT), and second WIT. Potassium-lowering medication used at admission, within $24 \mathrm{~h}$ prior to transplantation, during surgery, at the post-anaesthesia care unit (PACU), and within $48 \mathrm{~h}$ after transplantation was recorded. Use of calcineurin inhibitors and ACE inhibitors or angiotensin II receptor blockers at admission was also recorded. Frequency of dialysis within $24 \mathrm{~h}$ prior to transplantation and up to $48 \mathrm{~h}$ after transplantation was documented, as well as the time span between the last completed dialysis and transplantation. Hospital length of stay (LOS) was determined. Biochemical parameters collected for the recipient at the last moment immediately before transplantation included plasma potassium, sodium, chloride, corrected calcium, phosphate, albumin, and both haemoglobin and thrombocytes. Routine measurements were performed on the Roche Modular (Roche Ltd., Mannheim, Germany), while thrombocyte count and haemoglobin level were determined using a XN9000 (Sysmex, Etten-Leur, the Netherlands).

\section{Statistical Analyses}

IBM SPSS Statistics 25 (IBM Corp., Armonk, NY, USA) was used to perform statistical analyses. The relationship between pretransplant plasma potassium and the post-transplant occurrence of potassium-lowering interventions within $48 \mathrm{~h}$ was analyzed using multivariate logistic regression to test the association between 


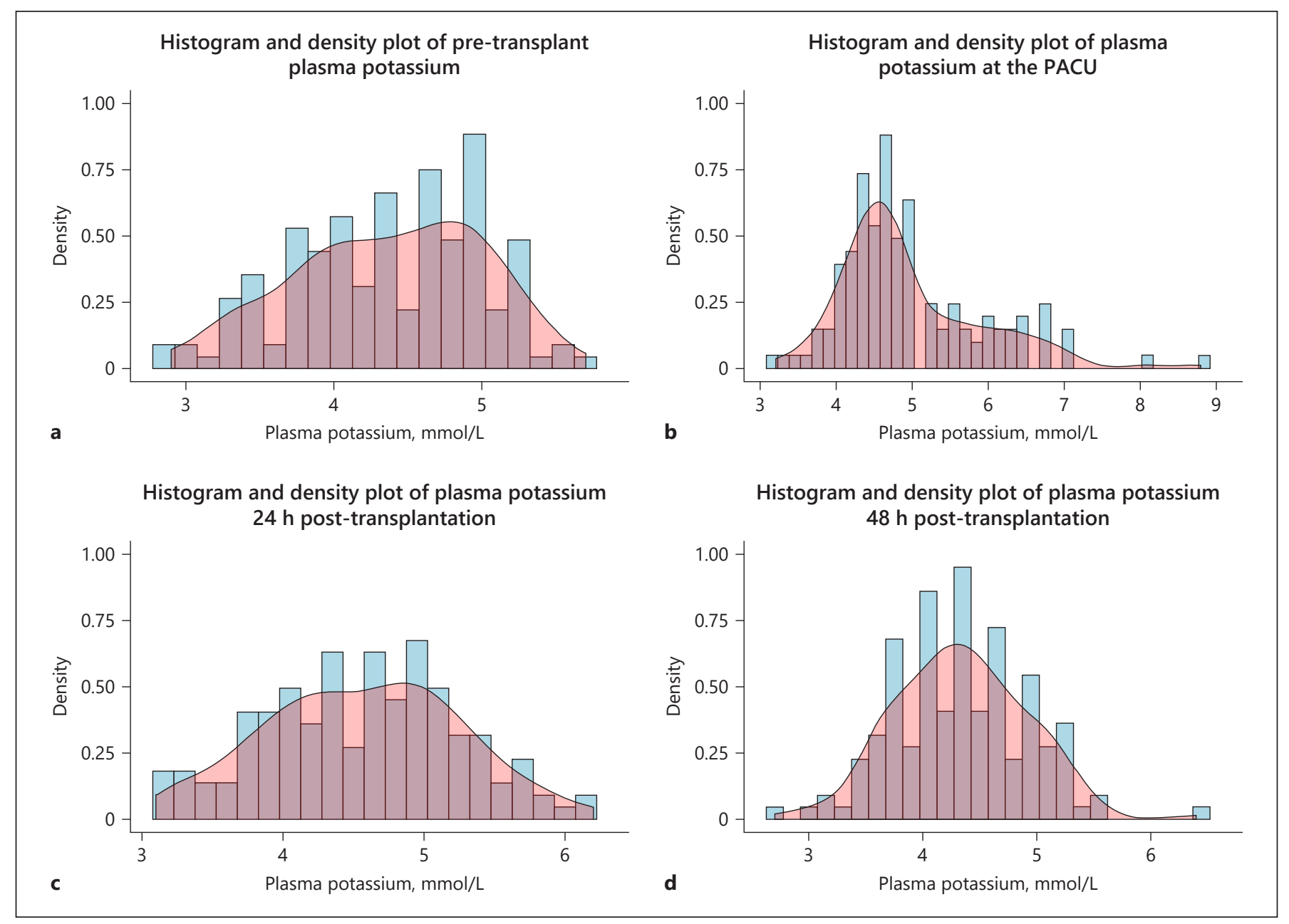

Fig. 1. Histogram and kernel-density plots of pre-transplant plasma potassium at the PACU (median $=4.4$ $[\mathrm{IQR}=3.9-4.9])(\mathbf{a})$, plasma potassium at the PACU (median $=4.7[\mathrm{IQR}=4.4-5.4])(\mathbf{b})$, plasma potassium $24 \mathrm{~h}$ after transplantation (median $=4.6[\mathrm{IQR}=4.1-5.1])(\mathrm{c})$, and plasma potassium $48 \mathrm{~h}$ after transplantation (me$\operatorname{dian}=4.3[\mathrm{IQR}=3.9-4.7])(\mathbf{d})$. PACU, post-anaesthesia care unit.

pre-transplant potassium and post-transplant potassium-lowering interventions, independent of other factors potentially influencing either of these variables. We first performed uni- and multivariate linear regression analyses to identify factors potentially influencing pre-transplant potassium and post-transplant potassium-lowering interventions. Subsequently, we performed multivariate logistic regression analysis to test the association between pre-transplant potassium and post-transplant potassium-lowering interventions. In univariate linear regression, all residuals were tested for normality, and all parameters with a $p$ value of $<0.05$ (two-tailed test) were selected for multivariate linear regression analysis. Subsequently, we performed multivariate logistic regression analysis with any post-transplant intervention as a dependent variable, again with all parameters that showed $p$ value $<0.05$ in the former multivariate linear regression. The variable time between last dialysis and transplantation was not included in multivariate analysis as this diminished the number of patients included $(n=$
47). Donor type was included in all models regardless of the $p$ value. All variables in both multivariate regression models were checked for multicollinearity by determining the variance inflation factor (VIF), as described by O'Brien [11].

Since pre-transplant plasma potassium was non-normally distributed, this variable was natural log transformed prior to linear regression analyses. A $p$ value of $<0.05$ was considered statistically significant. Furthermore, we tested for an interaction effect between pre-transplant plasma potassium and both donor type (deceased or living) and dialysis dependency before transplantation using post-transplant interventions as a dependent variable. We also checked for a potential interaction effect of pre-transplant plasma potassium and dialysis prior to transplantation. LOS was compared for patients with and without post-transplant potassium interventions using linear regression analysis. Finally, we tested for an association between pre-transplant potassium and LOS using regression analysis. 
Table 1. Baseline characteristics and corresponding $p$ values of the univariate linear regression analyses

\begin{tabular}{|c|c|c|c|c|c|}
\hline Recipient age, years & $55(45-63)$ & $55(44-63)$ & $56(47-66)$ & $58(50-64)$ & 0.064 \\
\hline Donor gender (female) & $72(48 \%)$ & $32(57 \%)$ & $21(38 \%)$ & $19(48 \%)$ & 0.59 \\
\hline Donor age, years & $57(48-64)$ & $57(47-64)$ & $57(48-66)$ & $54(46-63)$ & 0.31 \\
\hline Re-transplantation & $12(8 \%)$ & $5(8.9 \%)$ & $4(7.3 \%)$ & $3(7.5 \%)$ & 0.93 \\
\hline Cardiovascular co-morbidity & $53(35 \%)$ & $38(68 \%)$ & $36(66 \%)$ & $24(60 \%)$ & 0.40 \\
\hline Hypercholesterolaemia & $14(9 \%)$ & $4(7.1 \%)$ & $3(5.5 \%)$ & $7(18 \%)$ & 0.25 \\
\hline Hypertension & $69(46 \%)$ & $29(51 \%)$ & $21(38 \%)$ & $19(48 \%)$ & 0.73 \\
\hline Diabetes & $19(13 \%)$ & $7(13 \%)$ & $6(11 \%)$ & $6(15 \%)$ & 0.57 \\
\hline CIT, h & $3.0(2.4-11.0)$ & $11.6(3.2-15.4)$ & $3.2(2.4-12.7)$ & $3.4(2.4-11.9)$ & 0.001 \\
\hline Second WIT, min & $41(35-47)$ & $42(35-47)$ & $40(36-46)$ & $42(34-50)$ & 0.49 \\
\hline $\mathrm{K}^{+}$-lowering medication at admission & $29(19 \%)$ & $7(13 \%)$ & $12(22 \%)$ & $10(25 \%)$ & 0.37 \\
\hline ACE inhibitor or ARB at admission & $50(33 \%)$ & $20(36 \%)$ & $18(33 \%)$ & $12(30 \%)$ & 0.95 \\
\hline Calcineurin inhibitor at admission & $4(2.6 \%)$ & $3(5.4 \%)$ & $0(0 \%)$ & $1(2.5 \%)$ & 0.336 \\
\hline $\mathrm{K}^{+}$-lowering medication within $24 \mathrm{~h}$ prior to transplantation & $30(20 \%)$ & $1(1.8 \%)$ & $13(24 \%)$ & $16(40 \%)$ & $<0.001$ \\
\hline Chronic kidney disease, dialysis dependent & $111(74 \%)$ & $45(80 \%)$ & $38(69 \%)$ & $28(70 \%)$ & 0.15 \\
\hline Dialysis within $24 \mathrm{~h}$ prior to transplantation & $47(31 \%)$ & $22(39 \%)$ & $16(29 \%)$ & $9(23 \%)$ & 0.004 \\
\hline Time between last dialysis and transplantation, $\mathrm{h}$ & $10(4.0-17.0)$ & $5.5(3.0-12)$ & $16.0(4.2-17.0)$ & $16.0(6.0-17.5)$ & 0.001 \\
\hline Last plasma sodium before transplantation, $\mathrm{mmol} / \mathrm{L}$ & $141(138-142)$ & $141(137-142)$ & $141(138-142)$ & $141(139-143)$ & 0.86 \\
\hline Last plasma chloride before transplantation, $\mathrm{mmol} / \mathrm{L}$ & $98(95-101)$ & $99(95-101)$ & $101(96-105)$ & $99(97-107)$ & 0.039 \\
\hline Last haemoglobin before transplantation, $\mathrm{mmol} / \mathrm{L}$ & $7.5(7.0-8.0)$ & $7.5(6.9-8.0)$ & $7.4(6.7-7.9)$ & $7.1(6.2-8.0)$ & 0.34 \\
\hline
\end{tabular}

WIT, warm ischaemia time; CIT, cold ischaemia time; CI, confidence Interval; $\mathrm{K}^{+}$, potassium; ACE, angiotensin-converting enzyme; ARB, angiotensin II receptor blocker. Categorical variables are described as number (percentage), while continuous variables are described as median (first quartile to third quartile). $p$ value represents significance level of the univariate analysis with last plasma potassium before transplantation as the dependent variable.

\section{Results}

151 RTR were included in this study; 72 (48\%) received a living donor graft and $79(52 \%)$ received a deceased donor graft. Thirty three (42\%) of the deceased donors were DCD. The median age of recipients was 56 years (IQR $=47-64)$, and $47 \%$ was female. The median pre-transplant plasma potassium concentration was 4.4 $\mathrm{mmol} / \mathrm{l}(\mathrm{IQR}=3.9-4.9)$. A combined kernel-density plot with a histogram of the distribution of plasma potassium concentration at different time points is shown in Figure 1 . The median change of the plasma potassium concentration during surgery was $0.4 \mathrm{mmol} / \mathrm{L}(\mathrm{IQR}=-0.1$ to
1.2 ), with a maximum change of $\pm 4.7 \mathrm{mmol} / \mathrm{L}$. The median post-transplantation plasma potassium after 24 and $48 \mathrm{~h}$ was 4.6 (IQR = 4.1-5.1) and 4.3 (3.9-4.7), respectively. Within $48 \mathrm{~h}$ after kidney transplantation, 51 (33\%) patients received one or more interventions to resolve hyperkalaemia; 13 (25\%) patients received potassium-lowering medication, 31 (61\%) patients received dialysis, and 7 (14\%) patients received both. Forty three $(84 \%)$ of the patients undergoing one or more interventions had received a kidney from a deceased donor.

Baseline characteristics and corresponding $p$ values of the univariate linear regression analyses are shown in Table 1 . The results of the multivariate linear regression 
Table 2. Multivariate linear regression analysis with last plasma potassium before transplantation as the dependent variable

\begin{tabular}{lccc}
\hline & $\begin{array}{l}\text { Standardized } \\
\text { coefficient }\end{array}$ & $p$ value & VIF \\
\hline Donor type (deceased) & 0.010 & 0.95 & 4.2 \\
CIT, h & -0.18 & 0.22 & 4.1 \\
$\mathrm{~K}^{+}$-lowering medication within 24 h prior to transplantation & 0.33 & $<0.001$ & 1.2 \\
Dialysis within 24 h prior to transplantation & -0.12 & 0.15 & 1.2 \\
Last plasma chloride before transplantation, mmol/L & 0.006 & 0.95 & 1.3 \\
\hline
\end{tabular}

CIT, cold ischaemia time, $\mathrm{K}^{+}$, potassium; VIF, variance inflation factor.

Table 3. Multivariate logistic regression analysis with potassium-lowering intervention as the dependent variable

\begin{tabular}{lccc}
\hline & Exp B (CI) & $p$ value & VIF \\
\hline Donor type (deceased) & $11.5(4.6-29.2)$ & $<0.001$ & 1.1 \\
$\mathrm{~K}^{+}$-lowering medication within 24 h prior to transplantation & $0.6(0.2-1.8)$ & 0.34 & 1.2 \\
Last plasma potassium before transplantation, mmol/L & $2.2(1.1-4.4)$ & 0.018 & 1.2
\end{tabular}

$\mathrm{CI}$, confidence interval; $\mathrm{K}^{+}$, potassium; VIF, variance inflation factor.

analysis using the last available pre-transplant plasma potassium level are shown in Table 2. Donor type (deceased: beta $=0.25, p=0.002$ ), CIT (beta $=0.28, p=0.001$ ), potassium-lowering medication within $24 \mathrm{~h}$ prior to transplantation (beta $=0.40, p<0.001$ ), dialysis within $24 \mathrm{~h}$ prior to transplantation (beta $=0.23, p=0.004$ ), time between last dialysis and transplantation (beta $=0.45, p=$ 0.001 ), and last plasma chloride before transplantation (beta $=0.17, p=0.039$ ) showed a significant linear functional relationship with pre-transplant plasma potassium in univariate analysis. Upon multivariate linear regression analysis, including all correlated variables which were significant in univariate analysis, potassium-lowering medication within $24 \mathrm{~h}$ prior to transplantation was independently associated with pre-transplant potassium levels (beta $=0.33, p<0.001$ ).

Subsequently, we performed multivariate logistic regression analyses, adjusted for potential confounders identified during the aforementioned linear regression analyses, with post-transplant potassium-lowering interventions as the dependent variable. The results are shown in Table 3. Both donor type (deceased) and last plasma potassium before transplantation showed a significant association with potassium-lowering interventions, with odds ratios (95\% CI) of 11.5 (4.6-9.2) and 2.2 (1.1-4.4), respectively. No interaction between pre-transplant plasma potassium and both donor type $(p=0.16)$ and dialysis dependency before transplantation $(p=0.10)$ was found. Dialysis prior to transplantation showed no significant interaction with pre-transplant plasma potassium $(p=$ $0.57)$. All variables returned a VIF of $<5$; therefore, multicollinearity is low in the models.

Furthermore, a positive univariate association between pre-transplant plasma potassium and both the post-transplant plasma potassium at the PACU (beta = $0.212, p=0.013$ ) and $24 \mathrm{~h}$ post-transplant plasma (beta $=$ $0.183, p=0.026)$ was found. Post-transplant plasma potassium at the PACU was also a strong univariate predictor of post-transplant potassium-lowering interventions (odds ratio $=4.5$ [2.5-7.8]). Median LOS for patients who received post-transplant potassium-lowering interventions was significantly longer than that for patients with no need for post-transplant potassium-lowering interventions (LOS 18 [IQR: 15-23] and 16 [IQR: 15-16] days, respectively; $p=0.001$ ), also when corrected for donor type $(p=0.016)$. Pre-transplant potassium and LOS were significantly associated neither in a univariate linear regression analysis $(p=0.77)$ nor, after adjusting for donor type, in the multivariate linear regression analysis $(p=$ $0.357)$ 


\section{Discussion/Conclusion}

This pilot study is, to the best of our knowledge, the first to analyze the association between pre-transplant plasma potassium levels and the risk of requiring a potassium-lowering intervention within $48 \mathrm{~h}$ after kidney transplantation. Our study identified a higher pre-transplant plasma potassium as an independent risk factor for a potassium-lowering intervention, that is, dialysis or potassium-lowering medication, within $48 \mathrm{~h}$ after kidney transplantation.

The literature on the impact of hyperkalaemia around the time of kidney transplantation is scarce. Post-transplant hyperkalaemia caused by tacrolimus or cyclosporine has been studied widely $[12,13]$, but no studies regarding the relation between a pre-transplant plasma potassium and the occurrence of interventions to resolve hyperkalaemia among kidney transplantation patients have been performed previously. Dawwas et al. [14] performed a study considering pre-transplant potassium in liver transplantation patients and found that high pretransplant potassium levels are associated with greater risk of post-transplant renal dysfunction requiring shortor long-term renal support. DeFronzo et al. [15] addressed the mechanisms of hyperkalaemia following kidney transplantation within 3 months, but did not evaluate pre-transplant plasma potassium as a contributing factor.

Our finding of a positive linear functional relationship between pre-transplant plasma potassium and the occurrence of potassium-lowering interventions within $48 \mathrm{~h}$ after kidney transplantation may be explained by the higher starting point of potassium before transplantation. A rise in plasma potassium during surgery is known to be caused by different factors, such as reperfusion of ischaemic tissue and several types of medication, amongst others [7]. Pochineni and Rondon-Berrios [16] reviewed the most important factors that may drive hyperkalaemia in RTR, including renal tubular acidosis, insulin deficiency, or resistance, and medications, including trimethoprim (inhibiting epithelial sodium channels), calcineurin inhibitors (diminishing mineralocorticoid function and inhibiting the sodium-chloride co-transporter), and renin-angiotensin-aldosterone inhibitors (reducing potassium excretion). Bleeding and need for re-exploration may be other factors contributing to a higher potassium level in the post-transplant period [16]. Since patients with a higher plasma potassium before transplantation require a smaller increase in this concentration to develop hyperkalaemia, they might consequently have a higher risk for potassium-lowering interventions within
$48 \mathrm{~h}$ after kidney transplantation. Furthermore, we found that donor type was an important factor influencing the outcome. On the one hand, a possible explanation for the influence of donor type on both endpoints is the difference in quality of the donor graft. Gjertson and Cecka [17] showed a 7 and $24 \%$ rate of delayed graft function (DGF) for living unrelated renal grafts and deceased renal grafts, respectively. As dialysis is an important intervention to treat volume overload and hyperkalaemia in the context of DGF, it may be more common in patients with a deceased donor, independently of pre-transplant hyperkalaemia. Since our study is a retrospective study, the reason for dialysis (hyperkalaemia or hypervolaemia) could not fully be elucidated. On the other hand, confounding bias by indication may be another possible explanation. Physicians may be more cautious treating patients with a deceased donor as they seem more vulnerable, which could explain the higher occurrence of potassium-lowering interventions in patients with a deceased donor.

Our results suggest that per $1 \mathrm{mmol} / \mathrm{L}$ increase in pretransplant plasma potassium, RTR had a 2.2 times higher risk of requiring a potassium-lowering intervention after transplantation and that patients who received potassium-lowering interventions had a longer LOS compared to patients who did not. Whether potassium-lowering measures before transplantation might indeed contribute to less potassium-lowering interventions and have impact on long-term outcomes (morbidity and mortality) should be addressed in future studies. Furthermore, a cutoff level for pre-transplant plasma potassium needs to be determined to make the results useful in practice. As this is a retrospective study with a limited sample size, a cutoff threshold or range for correcting pre-transplant hyperkalaemia using a ROC curve could not be determined. Future research may determine if appropriate pre-transplant plasma potassium levels minimize the chances of encountering hyperkalaemia after transplantation. Avoiding the need for a potassium-lowering intervention after transplantation might contribute to a better quality of life in the short and long term. Both Taylor et al. [18] and Simons et al. [19] determined a negative relation between different side effects of medication and health-related quality of life in transplanted adolescents. Reducing the number of potassium-lowering interventions may minimize both discomfort for patients and healthcare costs. Furthermore, the new cation-exchange resins may also affect these outcomes since they are safe and effective for treatment of hyperkalaemia not only in CKD with an acceptable side-effect profile but also in RTR [20-23]. 
These may be a safer alternative for the use of sodium polystyrene sulfonate that is associated with colonic necrosis after renal transplantation [24, 25]. However, the influence of post-transplant potassium-lowering interventions on quality of life and the effectivity of precautionary measures was not the subject of this study and still needs to be verified in future studies.

Several limitations of this pilot study should be acknowledged, including its small sample size and its retrospective design. Furthermore, the effect of post-transplant potassium-lowering interventions in the long term was not taken into account. As a result, application of our research results in current practice is limited, and future studies are needed to confirm these results. Furthermore, arterial $\mathrm{pH}, \mathrm{HCO} 3-$, and use of potassium sparing diuretics were not considered as these were not structurally available before, during, or after transplantation. A limitation that also should be mentioned is that due to the lack of a specific threshold for treatment of post-transplant hyperkalaemia in our clinic, based on our study, it is difficult to make informed decisions on the appropriate interventions in a broader clinical context. Further research is required for the development of a widely applicable clinical threshold. It should also be noted that despite the fact that we adjusted for dialysis within $24 \mathrm{~h}$ prior to transplantation, remaining confounding by bias by indication cannot be excluded. Lastly, no data were available on the indication for post-transplant dialysis. Future prospective studies should collect such data to extend and validate our results.

In conclusion, this is the first study investigating the relation between pre-transplant plasma potassium and interventions to resolve hyperkalaemia. We show a positive linear functional relationship between pre-transplant plasma potassium and interventions to resolve hyperkalaemia within $48 \mathrm{~h}$ after kidney transplantation, support- ing the clinical importance of the pre-transplant plasma potassium in renal transplantation patients. As this is only a first step, further research is recommended to determine a cutoff level for pre-transplant plasma potassium that can be used in practice. Our findings should raise awareness of the pre-transplant plasma potassium level as a trigger to initiate a potassium-lowering intervention that could avoid post-transplant dialysis or medication.

\section{Statement of Ethics}

This study was approved by the institutional ethical review board (METc 2014/077). All procedures were conducted in accordance with the Declarations of Helsinki and Istanbul.

\section{Conflict of Interest Statement}

The authors have no conflicts of interest to declare.

\section{Funding Sources}

M.H.B. is supported by the Dutch Kidney Foundation (Grant No. CP1601) for research regarding potassium in renal transplantation patients.

\section{Author Contributions}

B.C.S.V. participated in the research design, collected the data, performed the statistical analysis, and drafted the article. S.P.B. and S.J.L.B. edited the article and participated in intellectual contributions. M.H.B. participated in the research design, statistical analysis, and revising the article. M.F.C.J. initiated the study, supervised data collection and progress of the research, participated in statistical analysis, and edited the article.

\section{References}

1 Ojo A. Addressing the global burden of chronic kidney disease through clinical and translational research. Trans Am Clin Climatol Assoc [Internet]. 2014;125(1):229-43.

2 Mucsi I, Kovacs AZ, Molnar MZ, Novak M. Co-morbidity and quality of life in chronic kidney disease patients. J Nephrol. 2008; 21(Suppl 13):991-5.

3 Global observatory on donation and transplantation [Internet]. [cited 2017 Apr 19]. Available from: http://www.transplant-observatory.org/data-charts-and-tables/chart/.

Pre-Transplant Potassium \& Hyperkalaemia Treatment after Kidney Transplantation
4 WHO|GKT1 Activity and Practices [Internet]. WHO. World Health Organization; 2013 [cited 2017 Apr 19]. Available from: http://www.who. int/transplantation/gkt/statistics/en/.

5 Eurotransplant - Statistics [Internet]. [cited 2018 Dec 16]. Available from: http://statistics. eurotransplant.org/index.php? search_ type=waiting+list\& search_organ=kidney\& search_region $=$ All+ET\&search_period $=2018$.

6 Luo J, Brunelli SM, Jensen DE, Yang A. Association between serum potassium and outcomes in patients with reduced kidney function. Clin J Am Soc Nephrol [Internet]. 2016 Jan 7 [cited 2019 Jul 8];11(1):90-100.
7 Ayach T, Nappo RW, Paugh-Miller JL, Ross EA Postoperative hyperkalemia. Eur J Intern Med [Internet]. 2015 Mar [cited 2019 Jul 8];26(2): 106-11. Available from: https://linkinghub. elsevier.com/retrieve/pii/S0953620515000291.

8 Xia VW, Ghobrial RM, Du B, Chen T, Hu K-Q, Hiatt JR, et al. Predictors of hyperkalemia in the prereperfusion, early postreperfusion, and late postreperfusion periods during adult liver transplantation. Anesth Analg [Internet]. 2007 Sep;105(3):780-5. 
9 Adwaney A, Randall DW, Blunden MJ, Prowle JR, Kirwan CJ. Perioperative PlasmaLyte use reduces the incidence of renal replacement therapy and hyperkalaemia following renal transplantation when compared with $0.9 \%$ saline: a retrospective cohort study. Clin Kidney J [Internet]. 2017 Dec;10(6):83844.

10 Weinberg L, Harris L, Bellomo R, Ierino FL, Story D, Eastwood G, et al. Effects of intraoperative and early postoperative normal saline or Plasma-Lyte $148^{\circledR}$ on hyperkalaemia in deceased donor renal transplantation: a doubleblind randomized trial. $\mathrm{Br} J$ Anaesth [Internet]. 2017 Oct 1;119(4):606-15.

11 O'brien RM. A caution regarding rules of thumb for variance inflation factors. Qual Quant [Internet]. 2007 Sep 11;41(5):673-90. Available from: http://link.springer.com/ 10.1007/s11135--006--9018--6.

12 Higgins R, Ramaiyan K, Dasgupta T, Kanji H, Fletcher S, Lam F, et al. Hyponatraemia and hyperkalaemia are more frequent in renal transplant recipients treated with tacrolimus than with cyclosporin. Further evidence for differences between cyclosporin and tacrolimus nephrotoxicities. Nephrol Dial Transplant. 2004;19(2):444-50.

13 Rehman S, Wen X, Casey MJ, Santos AH, Andreoni K. Effect of different tacrolimus levels on early outcomes after kidney transplantation. Ann Transplant [Internet]. 2014 Feb 7 [cited 2019 Jul 10];19:68-75. Available from: http://www.annalsoftransplantation.com/abstract/index/idArt/889858.
14 Dawwas MF, Lewsey JD, Watson CJ, Gimson AE, UK ILTA. The impact of serum potassium concentration on mortality after liver transplantation: a cohort multicenter study. Transplantation [Internet]. 2009 Aug 15 [cited 2019 Jul 8];88(3):40210.

15 DeFronzo RA, Goldberg M, Cooke CR, Barker C, Grossman RA, Agus ZS. Investigations into the mechanisms of hyperkalemia following renal transplantation. Kidney Int [Internet]. 1977;11(0085-2538 (Print)):357-65.

16 Pochineni V, Rondon-berrios H. Electrolyte and acid-base disorders in the renal transplant recipient. Front Med. 2018 Oct;5:1-11.

17 Gjertson DW, Cecka JM. Living unrelated donor kidney transplantation. Kidney Int. 2000; 58(2):491-9.

18 Taylor RM, Franck LS, Gibson F, Donaldson N, Dhawan A. Study of the factors affecting health-related quality of life in adolescents after liver transplantation. Am J Transplant. 2009;9(5):1179-88.

19 Simons LE, Anglin G, Warshaw BL, Mahle WT, Vincent RN, Blount RL. Understanding the pathway between the transplant experience and health-related quality of life outcomes in adolescents. Pediatr Transplant. 2008;12(2):187-93.

20 Bakris GL, Pitt B, Weir MR, Freeman MW, Mayo MR, Garza D, et al. Effect of patiromer on serum potassium level in patients with hyperkalemia and diabetic kidney disease. JAMA [Internet]. 2015 Jul 14;314(2):151. Available from: http://jama.jamanetwork.com/article. aspx?doi=10.1001/jama.2015.7446.
21 Weir MR, Bakris GL, Bushinsky DA, Mayo MR, Garza D, Stasiv Y, et al. Patiromer in patients with kidney disease and hyperkalemia receiving RAAS inhibitors. N Engl J Med [Internet]. 2015 Jan 15;372(3):211-21. Available from: http://www.nejm.org/doi/10.1056/ NEJMoa1410853.

22 Rattanavich R, Malone AF, Alhamad T. Safety and efficacy of patiromer use with tacrolimus in kidney transplant recipients. Transpl Int [Internet]. 2019 Jan;32(1):110-1. Available from: http://doi.wiley.com/10.1111/tri. 13369.

23 Lim MA, Sawinski D, Trofe-Clark J. Safety, effectiveness, and tolerability of patiromer in kidney transplant recipients. Transplantation [Internet]. 2019 Sep;103(9):e281-2. Available from: http://journals.lww.com/00007890-201909000--00037.

24 Pirenne J, Lledo-Garcia E, Benedetti E, West M, Hakim NS, Sutherland DE, et al. Colon perforation after renal transplantation: a single-institution review. Clin Transplant [Internet]. 1997 Apr;11(2):88-93.

25 Scott TR, Graham SM, Schweitzer EJ, Bartlett ST. Colonic necrosis following sodium polystyrene sulfonate (Kayexalate)-sorbitol enema in a renal transplant patient. Report of a case and review of the literature. Dis Colon Rectum [Internet]. 1993 Jun;36(6):607-9. 